\title{
Die Rechtsprechung des EuGH als bestimmender Faktor für die weitere Entwicklung der Koordinierung der Sozialversicherung innerhalb der Gemeinschaft
}

\author{
Sebastião Nóbrega PIZARRO
}

\section{Einführung}

1. Auch wenn es absurd ist, sollte man sich einmal vorstellen, dass es im EG-Vertrag keinen Artikel 177 (heute 234) gäbe, der die Zuständigkeit des Gerichtshofes bei Auslegung und Bewertung der Gültigkeit von Gemeinschaftsrecht regelt - und ich beschränke mich nur auf die an sich schon komplexen Vorschriften, die die Gesetzgebung zur sozialen Sicherheit der 27 Mitgliedstaaten regelt, welche ihrerseits vielfältig und komplex sind und oft unterschiedlichen Konzepten folgen. Dann ginge man zumindest das ernste Risiko ein, dass diese nicht einheitlich ausgelegt würden ${ }^{1}$.

Da dies im Widerspruch zum Integrationsprozess selbst steht, wäre eine noch schwerwiegendere Folge, dass schließlich der soziale Schutz in Frage gestellt würde, der den Unionsbürgern geschuldet wird, die ihr Recht auf Freizügigkeit im EU-Raum wahrgenommen haben oder wahrnehmen.

Nehmen wir auf der anderen Seite die Darlegung der Gründe, z.B. in der Verordnung Nr. 647/2005 zur Änderung der Verordnung 1408/71, bezüglich der Anwendung der Systeme der sozialen Sicherheit auf Arbeitnehmer und Selbständige sowie deren Familienangehörige, die innerhalb der Gemeinschaft $\mathrm{zu}$ und abwandern. Es mussten bestimmte Änderungen an der Verordnung vorgenommen werden, um namentlich die jüngste Rechtsprechung des Gerichtshofes der Europäischen Gemeinschaft zu berücksichtigen und dazu mussten Schlussfolgerungen aus verschiedenen Urteilen gezogen werden. Übrigens ist es auf diesem Gebiet so, dass die meisten Verordnungen mit Reformcharakter identische Erwägungen haben, während dies bei Verordnungen zur Abänderung früherer Verordnungen nur selten der Fall ist.

1 Der Art. 234 des Vertrages hatte ja gerade zum Ziel, einen Mechanismus zu schaffen, der die einheitliche Auslegung der gemeinschaftlichen Rechtsnormen sicherstellt, also einen Mechanismus, der wie man sehen wird, im Bereich der gemeinschaftlichen Koordinierungsregeln für soziale Sicherheit reichlich benutzt wurde. Anders ist es bei anderen multilateralen Koordinierungsregeln, z.B. werden beim Europäischen Abkommen über Soziale Sicherheit des Europarates von 1972 die Probleme der Auslegung durch Verhandlungen der streitenden Parteien gelöst oder, wenn die Differenzen für alle Vertragsparteien von Interesse sind und auf anderem Wege nicht beigelegt werden können, durch das Ministerkomitee, indem im Prinzip vom Präsidenten des Europäischen Gerichtshofes für Menschenrechte ein Schiedsrichter ernannt wird (vgl. Art. 71 des Europäischen Abkommens). 
Beide Feststellungen, eine imaginär, die andere real, zeigen die Bedeutung der Rechtsprechung des Gerichtshofes aus zwei Perspektiven, als zwei Seiten einer Medaille, nämlich als Entwicklung des Gemeinschaftsrechtes zur Koordinierung der Sozialversicherung ${ }^{2}$ und als effektiven Schutz der Unionsbürger und heutzutage auch, mit Anpassungen, der Bürger aus Drittstaaten.

2. Bevor wir das Herausragende dabei zeigen, möchte ich damit beginnen, einen lieben Freund und großen Meister auf diesem faszinierenden Gebiet, das sich 'Soziale Sicherheit für Wanderarbeitnehmer' nennt, zu zitieren.

In einem 1979 in einer belgischen Zeitschrift publizierten Artikel mit dem Titel: „L'influence de la Cour de Justice des Communautés Européennes sur la coordination internationale des législations de sécurité sociale“ (Der Einfluss des Gerichtshofes der Europäischen Gemeinschaften auf die internationale Koordinierung der Rechtsvorschriften über die soziale Sicherheit) hat der leider verstorbene Professor Guy Perrin gesagt: „es stellt sich also heraus, dass diese Rechtsprechung nicht nur eine Gemeinschaftsrechtsprechung ist, was sie auch sein soll, sondern sie ist auch eine grundsätzlich soziale und liberale Rechtsprechung. Sie ist nämlich insofern sozial, als sie dazu neigt, die für die Wanderarbeitnehmer günstigste Auslegung zu wählen, (...) [aber sie] ist auch liberal denn sie lehnt es ab, sich in den Wortlaut der Vorschriften einsperren zu lassen und entwickelt mitunter ihre eigene Logik sogar soweit, dass sie den Geist der Vorschrift bevorzugt, den sie ihnen beimisst" 3 .

Sozial und liberal, so nennt eine internationale Autorität auf diesem Gebiet die Rechtsprechung des Gerichtshofes. Attribute, die meines Erachtens auch nach 28 Jahren gut passen, wenn der Begriff liberal heute auch eine neoliberale Konnotation hat, was auf dem Gebiet der sozialen Sicherung allerdings nicht zutreffend ist.

3. Ich möchte vorausschicken, dass es natürlich nicht meine Absicht ist, in diesem Vortrag eine Analyse, auch nicht zusammenfassend, der Urteile des Gerichtshofes zu geben, mit dem Risiko, mich auf diesem steilen aber anregenden Weg, den die Gemeinschaftsrechtsprechung zur sozialen Sicherung darstellt, zu verlaufen.

Aus quantitativer Sicht - der eine reichhaltige qualitative Seite gegenübersteht muss man deutlich hervorheben, dass das Gericht seit dem berühmten Urteil UNGER ${ }^{4}$ aus dem Jahre 1964, dem ersten auf einem langen Weg, bis zu dem zuletzt veröffent-

2 Da der Begriff "Gemeinschaftsrecht zur Koordinierung der Sozialrechtsvorschriften der Mitgliedstaaten" in dieser oder anderer Form im Text sehr häufig vorkommt, wird aus Gründen der Vereinfachung die abgekürzte Bezeichnung Koordinierungsregelung oder Koordinierungssystem zur Sozialversicherung benutzt.

3 GUY PERRIN "L'influence de la Cour de Justice des Communautés européennes sur la coordination internationale des législations de sécurité sociale", Revue Belge de Sécurité Sociale, Bruxelles, janvier 1979, S.4. Übersetzung des Zitats aus dem Französischen.

4 Urteil UNGER, vom 19. März 1964, Rs 75/63. 
lichten Urteil STAMATELAKI aus $2007^{5}$ mehr als 550 Urteile auf dem Gebiet der sozialen Sicherung für Wanderarbeitnehmer gesprochen hat. Das ergibt im Mittel 13 Urteile pro Jahr, also mehr als ein Urteil pro Monat.

Man möchte fast sagen, dass der Gerichtshof die Existenz des Artikel 234 des Vertrags voll gerechtfertigt und seinen Beitrag zum Schutz der Wanderarbeitnehmer geleistet hat.

Wir werden jetzt zur Illustration und beispielhaft den Einfluss der Rechtsprechung des Gerichtshofes auf zwei Gebieten behandeln: einerseits die Begriffe Arbeitnehmer, Sozialversicherter und versicherte Person im Rahmen der Erweiterung des Personenkreises, auf den die Verordnungen zur Koordinierung der Sozialversicherung anzuwenden sind - natürlich immer mit dem Bezugspunkt der Freizügigkeit; und andererseits die Gewährung von Sachleistungen aus der Krankenversicherung im Ausland in den Fällen, wo dies auf Grund der nationalen Gesetzgebung von der Genehmigung durch den zuständigen Leistungsträger abhängt und zwar nach dem Decker-Urteil $1998^{6}$ angesichts der wirtschaftlichen Freiheiten, die mit der Freizügigkeit im Waren- und Dienstleistungsverkehr in Verbindung stehen ${ }^{7}$.

Der erste Punkt geht vor allem auf die Ursprünge dieser Rechtsprechung zurück, die diese tief prägen sollten, besonders in Bezug auf den festen und kohärenten Ausbau der Auslegung als Methode der Rechtsprechung; während der zweite ein stets aktuelles Thema ist und außerdem innerhalb des europäischen Sozialmodels mit der Gewährung von Gesundheitsleistungen im Gemeinschaftsraum zu tun hat.

Abschließend werden wir uns vorausblickend anschauen, welche Rolle die Rechtsprechung des Gerichtshofes eventuell bei der zukünftigen praktischen Anwendung der Verordnung Nr. 883/2004 zur Koordinierung der Systeme der sozialen Sicherung spielen kann. Darum muss man schon hier etwas zur Auslegungsmethode des Gerichtshofes sagen, das heißt, zur so genannten teleologische Methode der Auslegung.

\section{Die Methode der teleologischen Auslegung, wie sie vom Gerichtshof bei der Koordinierung der Systeme der sozialen Sicherung angewandt wird}

1. Die Bezeichnung der Regel als Koordinierung, anfänglich 'Soziale Sicherheit für Wanderarbeitnehmer' genannt, ergibt sich nicht aus dem Buchstaben des Artikels 51 des Vertrages (heute Art. 42). Die heutige Bezeichnung 'Koordinierung der Systeme der

5 Urteil STAMATELAKI, vom 19 April 2007, Rs C-444/05.

6 Urteil DECKER, vom 28. April 1998, Rs C-120/95.

7 Innerhalb des Textes abgekürzt 'wirtschaftliche Freiheiten'. 
sozialen Sicherheit' (Verordnung 883/2004) wurde in der Rechtsprechung des Gerichtes schon sehr viel früher als in der Gesetzgebung benutzt ${ }^{8}$.

Die Elemente, auf die sich der Gerichtshof in der Anfangsphase bei der Ausarbeitung seiner Auslegungsmethode gründete, ergaben sich aus zwei Hauptartikeln des Vertrages auf diesem Gebiet: Artikel 48 und 51 (heute 39 u. 42), die ihren Platz im Kapitel I zu den Arbeitskräften haben, welches seinerseits zu Titel III gehört, der der Freizügigkeit des Personenverkehrs gewidmet ist, welcher wiederum zum Zweiten Teil des Vertrages gehört, der den Grundlagen der Gemeinschaft gewidmet ist (heute mit dem Titel: Die Politiken der Gemeinschaft).

Erst später wurden andere Elemente in die Auslegungsmethode mit hinein genommen, die sich im Besonderen aus den Bestimmungen zur Freizügigkeit von Waren und Dienstleistungen ergeben. Dazu kamen die Elemente, die sich aus den Bestimmungen zur Unionsbürgerschaft ergeben.

Diese Referenz an die Grundlagen der Gemeinschaft hat den Gerichtshof dazu gebracht, die Auslegungsmethode „zu begründen“, insbesondere diejenigen, die in Anwendung des Artikels 234 eingesetzt wird, das heißt, im Rahmen der Zusammenarbeit zwischen dem Gerichtshof und den innerstaatlichen Gerichten, die mittels präjudiziellen Rückverweises als allgemeine Gerichte für das Gemeinschaftsrecht tätig sind.

Diese Methode mit der bedeutsamen Bezeichnung 'teleologische Methode' (aus griechisch telos - Ziel und logos - Lehre) orientiert sich in erster Linie an den Zielen des Vertrages, aus denen sich die Beweiskraft für die Kriterien herleitet, die den Gerichtshof auf der Suche nach Inhalt und Reichweite der zur Debatte stehenden Gemeinschaftsnorm führen. So wird durch die ausschließliche Zuständigkeit eine einheitliche Auslegung des Gemeinschaftsrechts gesichert.

Die Einheitlichkeit hat besondere Bedeutung bei den Koordinierungsregeln für die Sozialversicherung, welche durch Verordnungen bestimmt werden, die unterschiedliche Systeme bestehen lassen, denen unterschiedliche nationale Gesetzgebungen (Art. 42, Vertrag) und unterschiedlicher Institutionen entsprechen, gegen die die Leistungsempfänger „unmittelbare Ansprüche aufgrund der innerstaatlichen Rechtsvorschriften allein oder erforderlichenfalls in Verbindung mit dem in Artikel 51 des Vertrages vorgesehenen System der Zusammenrechnung der Versicherungszeiten"9 haben.

Diese Bestandteile oder „Grundlagen“, die vom Gerichtshof selbst in den ersten Urteilen in Auslegung der Verordnung Nr. 3 von 1958 zur Sozialen Sicherung der Wanderarbeitnehmer geschaffen wurden, können wie folgt zusammengefasst werden: 10

8 Siehe z.B. Urteil PETRONI, vom 21. Oktober 1975, Rs 24-75, Punkt 20, wo man lesen kann: „Artikel 46 Absatz 3 [der Verordnung Nr. 1408/71] ist eine Vorschrift zur Begrenzung von Kumulierungen. Der Rat darf auch bei der Ausübung der ihm in Artikel 51 verliehenen Befugnisse auf dem Gebiet der Koordinierung der mitgliedstaatlichen Systeme der sozialen Sicherheit ...".

9 Urteil DE MOOR, vom 5. Juli 1967, Rs 2-67, Abs. 10 der Entscheidungsgründe bezüglich des Fragenhintergrundes.

10 Die Hervorhebungen in diesem Beitrag stammen, besonders innerhalb der Zitate zur Rechtsprechung, vom Autor. 
Die Auslegung der Verordnungen erfolgt ausgehend von Artikel 39 und 51 des Vertrages, die seine Grundlage, Rahmen und Grenze sind.

Und so sagte das Gericht zu den Verordnungen der sozialen Sicherheit: „Die Grundlage, den Rahmen und die Grenzen der genannten Artikel, wie übrigens auch der gesamten Regelung, zu der sie gehören, bilden die Artikel 48 - 51 des Vertrages, deren Ziel die Herstellung der Freizügigkeit der Arbeitnehmer ist." Und somit lässt sich ihre Auslegung „von diesem grundlegenden Ziel ... nicht trennen“"11.

Bemerkt sei hier die logische, wenn nicht sogar chronologische Abfolge der drei Begriffe Grundlage, Rahmen und Grenze vom argumentativen Standpunkt aus. Das heißt, die Auslegung ist wirklich gemeinschaftlich und wird von den Bestimmungen des Vertrages befördert, vorgegeben und begrenzt.

Die Auslegung der Verordnungen muss ausgehend von der Freizügigkeit des Personenverkehrs als einem der Ziele des Vertrages geschehen.

„Da die Herstellung einer möglichst weitgehenden Freizügigkeit der Arbeitskräfte hiernach zu den 'Grundlagen' der Gemeinschaft gehört, stellt sie das Hauptziel des Artikels 51 dar und ist mithin für die Auslegung der in Anwendung dieses Artikels ergangenen Verordnungen maßgebend" 12 - ein leuchtendes Beispiel teleologischen Denkens in dem Sinne, dass jedwedes Recht einer Zielsetzung entspricht.

Die Auslegung der Verordnungen geht dahin, Benachteiligungen von Wanderarbeitnehmern zu verhindern und sie im Zweifelsfall möglicherweise sogar zu begünstigen.

Und in der Tat nimmt diese Freizügigkeit einen besonderen Platz unter den Rechten der Wanderarbeitnehmer ein. Darum wird der Gerichtshof gesagt haben: „Ein derartiges Ziel erfordert die Beseitigung von gesetzlichen Bestimmungen, die geeignet sind, die Wanderarbeitnehmer zu benachteiligen, und sich somit hemmend auswirken. Die vorerwähnten Artikel [48 bis 51] und die zu ihrer Durchführung getroffenen Maßnahmen sind daher [gerechterweise] im Zweifel in dem Sinne auszulegen, dass sie die Rechtsstellung der Wanderarbeitnehmer, insbesondere auf dem Gebiet der Sozialversicherung, vor Benachteiligungen schützen wollen. Dagegen stehen die genannten Vorschriften solchen innerstaatlichen Bestimmungen nicht entgegen, deren Anwendung dazu führt, dass die Wanderarbeitnehmer [zu ihren Gunsten] einen zusätzlichen Sozialschutz erhalten." 13 .

-Die Auslegung der Vorschriften muss so erfolgen, dass durch die Bestimmungen, die ja der Koordinierung und nicht der Harmonisierung der mitgliedstaatlichen Rechtsvorschriften zur sozialen Sicherheit dienen, keine zusätzlichen Differenzen zu denen hinzugefügt werden, die sich bereits aus der mangelnden Harmonisierung der nationalen Rechtsvorschriften ergeben.

11 Urteil HAGENBEEK, vom 13. Juni 1966, Rs 4/66, Absätze 5 u. 8 der Entscheidungsgründe.

12 Urteil UNGER, ebenda, Abs. 5 der Entscheidungsgründe.

13 Urteil NONNENMACHER, vom 19. Juni 1964, Rs 92/63, Abs. 13, 14 u. 15 der Entscheidungsgründe. 
So einen Fall gab es bei Artikel 73 der Verordnung 1408/71, der für die Zahlung der Familienbeihilfen zwei unterschiedliche Systeme festlegte, je nachdem, welchen Rechtsvorschriften die Wanderarbeitnehmer unterlagen: den französischen oder den Rechtsvorschriften aller anderen Mitgliedstaaten, womit das Gemeinschaftsrecht neue Ungleichheiten zu den bereits aus den einzelstaatlichen Rechtsvorschriften resultierenden Ungleichheiten hinzufügte.

$\mathrm{Zu}$ dieser Sache sagte der Gerichtshof, dass es notwendig sei: „darauf hinzuweisen, dass Artikel 51 [heute 42] EWG-Vertrag eine Koordinierung, nicht aber eine Harmonisierung der Rechtsvorschriften (...) vorsieht. Artikel 51 lässt also Unterschiede zwischen den Systemen der Sozialen Sicherheit der Mitgliedstaaten (...) bestehen. Daraus folgt, dass das (...) [Gemeinschaftsrecht] keine Unterschiede einführen darf, die zu denen hinzutreten, die sich bereits aus der mangelnden Harmonisierung der nationalen Rechtsvorschriften ergeben." 14

Die Auslegung der Gemeinschaftlichen Rechtsvorschriften verbietet es nicht, dass durch innerstaatliche Gesetze den Wanderarbeitnehmern noch günstigere Leistungen gewährt werden.

Die Verordnung 1408/71 stellt für den Gerichtshof ,zu diesem Zweck ... eine Gesamtheit von Vorschriften auf, die sich insbesondere auf das Verbot der Diskriminierung aufgrund der Staatsangehörigkeit oder des Aufenthaltsortes sowie auf die Aufrechterhaltung der Ansprüche gründen, die der Arbeitnehmer nach dem System oder den Systemen der sozialen Sicherheit, die für ihn gelten oder gegolten haben, erworben hat. Es hieße demnach, über dieses Ziel hinauszugehen und sich gleichzeitig außerhalb der Zweckbestimmung und des Rahmens des vorgenannten Artikels 51 zu stellen, legte man die Verordnung Nr. 1408/71 so aus, dass sie innerstaatlichen Rechtsvorschriften verbietet, dem Arbeitnehmer einen weitergehenden sozialen Schutz zu gewähren, als sich aus der Anwendung dieser Verordnung ergibt."15.

Die Auslegung der Verordnungen darf die von den Wanderarbeitnehmern errungenen Rechte, die sich aus der ausschließlichen Anwendung einer einzelstaatlichen Rechtsvorschrift ergeben, nicht in Frage stellen.

Anders gesagt, muss das so genannte Prinzip der Unantastbarkeit der erworbenen Rechte als Schlussfolgerung aus den Artikeln 39 und 42 des Vertrages respektiert werden, deren Zweck es ihrerseits ist, den freien Personenverkehr zu sichern und zu fördern.

Wie RAEPENBUSCH bei der Einschätzung dieses Prinzips hervorhebt, „cet objectif ne serait pas atteint si l'application des règlements avait pour résultat de supprimer ou de réduire les avantages de sécurité sociale qu'un travailleur tiendrait de la seule législa-

14 Urteil PINNA, vom 15. Januar 1986, Rs 41/84, Rdnr. 20 u. 21.

15 Urteil JORDENS-VOSTERS, vom 10. Januar 1980, Rs 69/79, Rdnr. 11. 
tion d'un État membre et de placer ainsi le travailleur migrant dans une situation moins favorable que celle que lui garantissent les législations nationales" 16

Mit anderen, bereits vor mehr als dreißig Jahren vom Gerichtshof im berühmten Urteil PETRONI von 1975 gesprochenen Worten würde „,der Zweck der Artikel 48 bis 51 [heute 39 und 42] (...) verfehlt, wenn die Arbeitnehmer, die von ihrem Recht auf Freizügigkeit Gebrauch gemacht haben, Vergünstigungen der sozialen Sicherheit verlören, die ihnen jedenfalls die Rechtsvorschriften eines einzigen Mitgliedstaats sichern."17

Bei der Auslegung der Verordnungen muss man in den Bestimmungen danach Ausschau halten, wo sich die Grundprinzipien der Koordinierungsregeln und somit das Prinzip der weitesten Gleichbehandlung der Bürger der Mitgliedsstaaten widerspiegeln, was sowohl unmittelbare, mittelbare oder verdeckte Diskriminierung verbietet und außerdem muss man, wenn notwendig, die Sachlage in einem Mitgliedstaat an die in einem anderen Mitgliedstaat bestehende Sachlage anpassen.

Am spürbarsten für die Wanderarbeitnehmer ist das Prinzip der Aufrechterhaltung von Rechten, insbesondere von erworbenen Rechten und das Prinzip der Gleichbehandlung. Hierbei hat das Prinzip der Festlegung der anwendbaren Rechtsvorschriften eine instrumentelle Funktion, die zwar vorausgeht, aber in dem Maße bestimmend ist, da sie in der konkreten Situation die einzelstaatlichen Gesetzesvorschriften auswählt, die zwingend für den Leistungsempfänger gelten.

Zum ersten Prinzip hat sich der Gerichtshof explizit und mit der Forderung nach Verzicht auf Klauseln der Wohnsitzerfordernis oder der Einredbarkeit von Territorialklauseln gegen den Leistungsempfänger sehr klar geäußert als er sagte: „Vorschriften (...), die Ausnahmen vom Grundsatz der Exportierbarkeit von Leistungen der sozialen Sicherheit vorsehen (...) sind eng auszulegen." 18

Bezüglich des zweiten Prinzips, aus dem sich alle anderen Prinzipien ableiten, die das System der Koordinierungsregeln bilden, vertritt der Gerichtshof die Meinung, dass es auch in den Fällen, wo die Leistungsempfänger anscheinend gleichbehandelt werden, Diskriminierung wegen der Staatsangehörigkeit geben kann, nämlich dort, wo man durch Anwendung anderer Kriterien, z.B. Wohnsitz der Familie, zum gleichen Ergebnis kommt und „dass der Gleichbehandlungsgrundsatz nicht nur offenkundige Diskriminierungen aufgrund der Staatsangehörigkeit, sondern auch alle verschleierten Formen der Diskriminierung verbietet, die mit Hilfe der Anwendung anderer Unterscheidungsmerkmale tatsächlich zu demselben Ergebnis führen"19.

Und bezüglich der Anpassung an die Sachlage gilt: „Die zuständige Einrichtung eines Mitgliedstaats hat nach den Artikeln 8a, 48 und 51 EG-Vertrag (nach Änderung

16 S.V. RAEPENBUSCH "La Sécurité sociale des travailleurs européens, Principes directeurs et grands arrêts de la Cour de Justice des Communautés européennes », éditions de Boeck, Université, Bruxelles, 2001, p.142.

17 Urteil PETRONI, ebenda, Rdnr. 13.

18 Urteil JAUCH, vom 8. März 2001, Rs C-215/99, Rdnr. 21.

19 Urteil PINNA, ebenda, Rdnr. 23. 
jetzt Artikel 18 EG, 39 EG und 42 EG) Kindererziehungszeiten, die eine zur Zeit der Geburt des Kindes als Grenzgänger in diesem Mitgliedstaat beschäftigte und in einem anderen Mitgliedstaat wohnhafte Person in letzterem zurückgelegt hat, für die Gewährung der Altersrente wie im Inland zurückgelegte Zeiten anzurechnen." 20

Bei der Auslegung der Verordnungen wird berücksichtigt, ob es sich bei einer Leistung um eine Leistung der sozialen Sicherheit handelt und worin das Wesen der Leistung, wie Zweck und Voraussetzung für die Gewährung, besteht - unabhängig davon ob die Leistung als solche nach nationalem Recht eines Mitgliedsstaates eine Leistung der sozialen Sicherheit ist - sofern es sich, einschränkend, um ein Risiko im sachlichen Geltungsbereich der gleichen Verordnungen handelt.

$\mathrm{Da}$ es um die koordinierte Geltung einzelstaatlicher Systeme der sozialen Sicherheit geht, fühlte sich der Gerichtshof logischerweise bemüht, die durch dieses System zu diesem Zweck gewährten Leistungen einheitlich und genau zu beschreiben, also festzustellen, wann eine Leistung von den Verordnungen erfasst oder ausgeschlossen werden soll. Damit wird jedem einzelnen Mitgliedstaat die Definition „seines“ Feldes der sachlichen Anwendbarkeit entzogen.

Somit kann ,eine Leistung (...) dann als Leistung der sozialen Sicherheit betrachtet werden, wenn sie erstens den Empfängern ohne jede auf Ermessen beruhende individuelle Bedürftigkeitsprüfung aufgrund eines gesetzlichen Tatbestands gewährt wird und sich zweitens auf eines der in Artikel 4 Absatz 1 der Verordnung Nr. 1408/71 ausdrücklich aufgezählten Risiken bezieht." 21

Die Auslegung der Koordinierungsregeln zur sozialen Sicherheit im Sinne des Ausschlusses eines Geltungsbereiches, insbesondere bestimmter Leistungen, also im Sinne von Nichtgeltung, darf die Mitgliedsstaaten nicht davon entbinden, sich zu vergewissern, dass keine andere Vorschrift des Gemeinschaftsrechtes gelten.

Eine Leistung, die theoretisch eine Leistung der sozialen Sicherheit im oben benannten Sinne sein kann, muss ,,angesichts des weiteren Spielraums, über den der Rat (...) verfügt" 22 bei der Festsetzung des sachlichen Geltungsbereich der Koordinierungsregel mit erfasst werden und im Lichte der Verordnung 1612/68 über den freien Personenverkehr der Arbeitnehmer innerhalb der Gemeinschaft geprüft werden.

Der Gerichtshof sagt: „Der Ausschluss der besonderen Geburts- und Adoptionsbeihilfen vom Anwendungsbereich der Verordnung Nr. 1408/71 (...) befreit jedoch (...) die Mitgliedstaaten nicht von der Verpflichtung, sich zu vergewissern, dass keine andere

20 Urteil ELSEN, vom 23. November 2000, Rs C-135/99, Tenor.

21 Urteil MEINTS, vom 27. November 1997, Rs C-57/96, Rdnr. 23 u. 24.

22 Urteil GISLAIN LECLERE U. ALINA DEACONESCU, vom 31. Mai 2001, Rs C-43/99, Rdnr. 29 u. 31. Der ,, weitere Spielraum des Gerichtshofes “, würden wir hinzufügen, darf jedoch nicht soweit ausgedehnt werden, dass Artikel 42 des Vertrages in Frage gestellt wird, der grundsätzlich die Leistungen umfasst, die von den "verschiedenen einzelstaatlichen Gesetzesvorschriften" vorgesehen sind, jedenfalls mindestens jene Leistungen die einem der in der Koordinierungsverordnung ausdrücklich aufgezählten Risiken entsprechen. 
Vorschrift des Gemeinschaftsrechts, insbesondere der Verordnung Nr. 1612/68, der Aufstellung einer Wohnortvoraussetzung entgegensteht.، ${ }^{\text {22 }}$

Und falls hier etwas entgegensteht, kann es im Gegenteil auf Grund des Prinzips der Gleichbehandlung und der Nichtdiskriminierung wegen Staatsangehörigkeit zur Gewährung einer Leistung der sozialen Sicherheit kommen, auch wenn man sich damit an den Rand der Koordinierungsregeln begibt.

2. Wenn es stimmt, dass die Auslegung der Normen zu Koordinierung der Sozialversicherung durch den Gerichtshof anfänglich von der Durchsetzung des Zieles 'Freizügigkeit der Arbeitnehmer' und von den Grundprinzipien der Koordinierung der Sozialversicherung „,nicht [zu] trennen“ 23 war, dann bilden in jüngerer Zeit auch andere Freiheiten und andere Prinzipien des Vertrags die „Grundlage“ und den „Rahmen“23 - aber in gewisser Weise schon nicht mehr die Grenzen (so die erweiterte Betrachtung der Gründe und Ziele) - für die Rechtsprechung.

Es geht zum Beispiel um wirtschaftliche Freiheiten, hier besonders um die Dienstleistungsfreiheit im Gesundheitswesen und um das Prinzip der Unionsbürgerschaft. Das bedeutet:

Die Auslegung der Verordnungen darf sich also nicht auf die traditionelle Prüfung der Übereinstimmung mit den als Rechtsgrundlage dienenden Grundbestimmungen des Vertrages beschränken, sondern muss, wenn notwendig, die auszulegende Bestimmung auch im Zusammenhang mit anderen, ebenfalls grundlegenden Bestimmungen sehen.

Der Gerichtshof sagte: „Der Umstand, dass eine nationale Maßnahme möglicherweise einer Bestimmung des abgeleiteten Rechts - hier dem Artikel 22 der Verordnung Nr. 1408/71 - entspricht, hat nicht zur Folge, dass sie nicht an den Bestimmungen des EGVertrags zu messen wäre" oder genauer an den Artikeln 59 und 60 (heute 49 und 50); auf der anderen Seite „lässt das Gemeinschaftsrecht die Zuständigkeit der Mitgliedstaaten zur Ausgestaltung ihrer Systeme der sozialen Sicherheit unberührt (...). Gleichwohl müssen die Mitgliedstaaten (...) bei der Ausübung dieser Befugnis das Gemeinschaftsrecht beachten." 24

Die Auslegung der Verordnungen zur sozialen Sicherung muss derart erfolgen, dass alle Bürger der EU, die ihren Wohnsitz in einem Mitgliedstaat haben, erfasst werden und als solche ein Recht auf Nichtdiskriminierung im Verhältnis zu den Staatsangehörigen des Aufnahmestaates haben.

Der Status 'Unionsbürger' hat das Zeug, grundlegender Status aller Staatsangehörigen der Mitgliedstaaten zu werden, denn die Bürger der Union unterliegen, so sie hier ihren Wohnsitz haben, den Rechten und Pflichten aus dem Vertrag (Artikel 17). Zu diesen Rechten gehört das Recht auf Nichtdiskriminierung wegen der Staatszugehörigkeit auf den Feldern auf denen der Vertrag anwendbar ist, hier also bei den Koordinierungsregeln für die Sozialversicherung. Und in der Tat sagte der Gerichtshof, dass „,ich ein

23 Vgl. oben, Urteil HAGENBEEK.

24 Urteil DECKER, ebenda, Rdnr. 27, 21 und 23. 
Unionsbürger, der sich (...) rechtmäßig im Gebiet des Aufnahmemitgliedstaats aufhält, in allen vom sachlichen Anwendungsbereich des Gemeinschaftsrechts erfassten Fällen auf Artikel 6 des Vertrages berufen [kann], und zwar auch in dem Fall, dass dieser Staat die Gewährung einer Leistung [der Sozialversicherung], die jeder Person zusteht, die sich rechtmäßig in diesem Staat aufhält, verzögert oder verweigert" ${ }^{\star 25}$.

Die Auslegung der gemeinschaftlichen Rechtsvorschriften muss auch solche Fälle berücksichtigen, die ausnahmsweise entweder das Vorhandensein von Hindernissen für die Grundfreiheiten des Vertrags oder die Einschränkung der Grundprinzipien der Koordinierungsregeln rechtfertigen, falls diese Beschränkungen objektiv und angemessen sind, und es darf auch nicht ausgeschlossen werden, dass eine Auslegung eventuell zu Anpassungen in den innerstaatlichen Systemen der sozialen Sicherung führt.

„Es steht fest, dass eine nationale Regelung (...), die einige Staatsangehörige allein deswegen benachteiligt, weil sie ihre Freiheit, sich in einen anderen Mitgliedstaat zu begeben und sich dort aufzuhalten, wahrgenommen haben, eine Beschränkung der Freiheiten darstellt, die Artikel 18 EG jedem Unionsbürger verleiht (...). Eine solche Beschränkung kann nach Gemeinschaftsrecht nur dann gerechtfertigt sein, wenn sie auf objektiven (...) Erwägungen des Allgemeininteresses beruht, die in einem angemessenen Verhältnis zu dem mit dem nationalen Recht rechtmäßigerweise verfolgten Zweck stehen. (...) Eine Maßnahme ist dann verhältnismäßig, wenn sie zur Verwirklichung des verfolgten Zieles geeignet ist und nicht über das hinausgeht, was zu dessen Erreichung notwendig ist." 26

Auf der anderen Seite, und wenn wir über die Anpassungen sprechen, die in den mitgliedstaatlichen Gesetzgebungen bezüglich der Arbeitslosenunterstützung vorgenommen werden mussten, genauer gesagt bezüglich der Auszahlung auf dem Territorium eines anderen Mitgliedstaates, hat der Gerichtshof bestätigt, dass „die Verwirklichung der durch den Vertrag garantierten Grundfreiheiten die Mitgliedstaaten unvermeidlich [verpflichtet], einige Anpassungen in ihren nationalen Systemen der sozialen Sicherheit vorzunehmen, ohne dass dies als Eingriff in ihre souveräne Zuständigkeit in dem betreffenden Bereich angesehen werden könnte.“27

Das ist natürlich vom Standpunkt des Systementwurfs interessant, denn die Verordnungen schaffen Regeln zur Koordinierung und nicht zur Harmonisierung und ändern also die einzelstaatlichen Gesetzesvorschriften nicht. Wenn man nun zulässt, dass diese bisweilen Anpassungen erfahren - was mit Sicherheit noch keine Harmonisierung ist -, so wird doch in gewisser Weise der Begriff Koordinierung im engen Sinne überschritten.

25 Urteil SALA, vom 12. Mai 1998, Rs C-85/96, Rdnr. 63.

26 Urteil DE CUYPER, vom 18. Juni 2006, Rs C-406/04, Rdnr. 39, 40 u. 42.

27 Urteil MÜLLER-FAURÉ, vom 13. Mai 2003, Rs C-385/99, Rdnr. 102. 
Alle diese Bestandteile, Kriterien und Orientierungen - für die hier bedeutende Beispiele $^{28}$ zur Illustration der richterlichen Denkweise gebracht wurden - wurden den Prinzipien und den grundlegenden Zielen des Vertrages vom Gerichtshof selbst entnommen.

III. Der Einfluss der Rechtsprechung des EuGH auf den persönlichen Anwendungsbereich der Koordinierungsregel - der lange Weg von den Arbeitnehmern und Gleichgestellten bis zu den Staatsangehörigen der Mitgliedstaaten und bis zur Ausweitung auf Drittstaatenangehörige

1. Der persönliche Geltungsbereich der Verordnung 3/1958 zur sozialen Sicherung der Wanderarbeitnehmer ist bei diesen von einer Kategorie 'Arbeitnehmer oder Gleichgestellte' ausgegangen ohne diese Kategorie jedoch genau zu definieren, und hat somit dem Gerichtshof breiten Raum für die richterliche Auslegung gelassen, welcher auf der Grundlage des Gemeinschaftsrechtes ausgefüllt und ausgestaltet werden sollte, auch entgegen manchmal abweichender Kriterien innerstaatlicher Rechtsvorschriften, also „zugunsten einer einheitlichen Auffassung, die mehr im Einklang mit den Anforderungen eines europäischen Rechtsstatus hinsichtlich des Schutzes der Wanderarbeitnehmer" 29 .

Bei der ersten einer langen Serie von grundlegenden Fragen wurde der Gerichtshof, wie wir beim Urteil UNGER gesehen haben, vor die Frage gestellt, herauszufinden, „ob sich der Begriff 'ein Arbeitnehmer oder ihm Gleichgestellter' (...) nach dem Recht der einzelnen Mitgliedstaaten bestimmt oder einen übernationalen Inhalt hat, mit welcher Wendung offensichtlich das Gemeinschaftsrecht gemeint ist“"30.

Der Antwort wurde eine konsistente Argumentation vorangestellt: „Wäre die Bestimmung dieses Begriffes dem innerstaatlichen Recht überlassen worden, so wäre jeder

28 Die Rechtsprechung in Sachen VAN MÜNSTER (Urteil vom 5. Oktober 1994, Rs C-165/91) und ENGELBRECHT (Urteil vom 26. Oktober 2000, Rs C-252/97) kann ebenfalls im Sinne der teleologischen Methode gesehen werden, auch wenn es hier darum geht, dass der innerstaatliche Richter gezwungen ist, innerstaatliche Rechtsvorschriften zur Sozialversicherung "in Übereinstimmung mit den Anforderungen des Gemeinschaftsrechts auszulegen". Falls dies unmöglich sein sollte, hat dieser Richter, um die vollständige Anwendung dieses Rechtes sicherzustellen, sogar "die Pflicht (...) nach Artikel 5 EG-Vertrag", "notfalls jede Bestimmung unangewandt [zu lassen], deren Anwendung im konkreten Fall zu einem gemeinschaftsrechtswidrigen Ergebnis führen würde" (Rdnr. 39, 38 u. 40 Urteil ENGELBRECHT). Wenn man nun weiß, dass man vor einem "gemeinschaftsrechtswidrigem Ergebnis" steht, (z.B. die Situation, die aus einem nichtkonformen Verständnis der Koordinierungsregeln entsteht, was einen Arbeitnehmer dazu bringen könnte, auf die Ausübung seines Rechtes auf Freizügigkeit zu verzichten) kann man schließlich zum Gerichtshof gehen und eine Auslegung der Koordinierungsregelung verlangen.

29 GUY PERRIN, ebenda, S. 4.

30 Urteil UNGER, Abs. 1, 7, 10, 15 u. 26 der Entscheidungsgründe. 
Staat in der Lage, den Inhalt des Begriffes 'Wanderarbeitnehmer' Veränderungen zu unterwerfen und bestimmten Personengruppen nach Belieben den Schutz des Vertrages zu entziehen.“ Das Ergebnis davon wäre dann, dass die „Artikel 48 bis 51 jeglicher Bedeutung beraubt und die vorerwähnten Ziele des Vertrages ernsthaft gefährdet ${ }^{\star 30}$ wären.

Die Schlussfolgerung des Gerichtshofes hätte nicht klarer und konsequenter sein können und hat das Primat des Gemeinschaftsbegriffes festgeschrieben: „Nach alledem ist der Begriff 'ein Arbeitnehmer oder ihm Gleichgestellter' vom Gemeinschaftsrecht her zu verstehen. Er erstreckt sich auf alle Personen, die in dieser Eigenschaft, gleichviel unter welcher Bezeichnung, von den verschiedenen Systemen des innerstaatlichen Sozialversicherungsrechts erfasst werden. ${ }^{630}$.

In dem Maße, wie der Akzent immer stärker auf Sozialversicherungsrecht statt auf Arbeitsrecht gelegt wurde, sollte der letzte Teil der Antwort von nun an Ausgangspunkt der schrittweisen Erweiterung des persönlichen Geltungsbereiches der Gemeinschaftsnormen zur sozialen Sicherung werden.

Und der Gerichtshof hat im gleichen Urteil UNGER alle diejenigen mit eingeschlossen, die streng genommen zwar nicht als Wanderarbeitnehmer angesehen werden können, (und man muss daran erinnern, dass der Titel der Verordnung Nr. 3 gerade die soziale Sicherung der Wanderarbeitnehmer zum Gegenstand hatte) die sich aber innerhalb des Gemeinschaftsterritoriums bewegen, ,gleichviel welches der Grund [ihres] Auslandsaufenthaltes ist ${ }^{430}$.

2. Auf der anderen Seite ist es für den Umfang des Begriffs Arbeitnehmer, so wie er vom Gerichtshof ausgelegt wurde, wenig relevant, ob der Betreffende „nun eine Erwerbstätigkeit ausübt oder nicht“"31; entscheidend ist, ob derjenige ,die Versicherteneigenschaft nach den für die soziale Sicherheit geltenden Rechtsvorschriften eines oder mehrerer Mitgliedstaaten besitzt" und falls die betreffende Person eine Berufstätigkeit ausübt, muss die Eigenschaft Arbeitnehmer , unabhängig vom zeitlichen Umfang ihrer Beschäftigung“32 gelten, also selbst wenn es sich um eine Teilzeitbeschäftigung handelt.

Außerdem ,ist eine Person dann als Arbeitnehmer (...) anzusehen, wenn sie die materiellen Voraussetzungen erfüllt, die von dem für sie geltenden System der sozialen Sicherheit objektiv festgesetzt worden sind, auch wenn die für den Anschluss an dieses System erforderlichen Schritte nicht unternommen wurden"33, also selbst, wenn sie aus Gründen, die die Arbeitgeberseite zu vertreten hat, nicht wirksam sozialversichert ist.

Die für eine Erweiterung des Leistungsempfängerkreises günstige Auffassung in den gemeinschaftlichen Rechtsvorschriften zur Sozialversicherung wurde durch die richterliche Einführung des Begriffes 'Arbeitnehmern Gleichgestellte' noch weitergeführt.

31 Urteil PIERIK, vom 31. Mai 1979, Rs C-182/78, Rdnr. 4.

32 Urteil KITS VAN HEIJNINGEN, vom 3. Mai 1990, Rs C-2/89, Rdnr. 10.

33 Urteil MOUTHAAN, vom 15. Dezember 1976, Rs C-39/76, Rdnr. 10. 
In der Rechtssache DE CICCO aus 1968 ging es um einen selbständigen Handwerker, der also nicht unter die Kategorie Arbeitnehmer zu fassen war. Der Gerichtshof hat dazu festgestellt: „Eine solche Gleichstellung liegt stets dann vor, wenn nach dem Recht eines Mitgliedstaates die Vorschriften über ein allgemeines Sozialversicherungssystem auf eine Personengruppe erstreckt werden, die nicht zu den Arbeitnehmern (...) gehört; hierbei kommt es nicht darauf an, welcher Mittel und Wege sich der staatliche Gesetzgeber zu diesem Zweck bedient"34. Diese Gleichsetzung wurde durch das Urteil JANSSEN vom 27. Oktober 1971, Rs C-23/71, bestätigt.

Man musste jedoch noch bis zur Verordnung 1390/81 warten, bis die Koordinierungsregel - durch hilfsweisen Rückgriff auf Artikel 235 (heute 308) - auf Selbständige ausgedehnt wurde.

Interessant ist jedoch, dass in der 6. Erwägung dieser Verordnung festgestellt wird, dass sich die Verordnung 1408/71, auch wenn sie für Arbeitnehmer gilt, auch schon auf bestimmte Kategorien von selbständig Tätigen erstreckte, was als impliziter Bezug auf die Rechtsprechung des Gerichtshofes zu sehen ist und somit als Bezug auf seinen Einfluss auf die Entwicklung des persönlichen Geltungsbereiches der Koordinierungsregeln.

3. Im Rahmen einer auf der einen Seite echten Freizügigkeit des Personenverkehrs (und nicht nur für Arbeitnehmer) in einem Raum ohne innere Grenzen (Art. 14 des Vertrages), in dem diese Freiheit garantiert wird und auf der anderen Seite einer Unionsbürgerschaft, mit der die Staatsangehörigen die im Vertrag (Art. 17 u. 18) vorgesehenen Rechte und Pflichten haben, wäre es schwer möglich, wenn nicht gar unmöglich, dass die Erweiterung des persönliches Geltungsbereiches der gemeinschaftlichen Rechtsvorschriften zur sozialen Sicherung bei den selbständig Tätigen stehen bliebe.

In der Tat gab es weiterhin drei Kategorien von Personen, die sich nicht auf die Verordnungen berufen konnten: die zu einem Sondersystem gehörenden Beamten; die Studenten als solche (und nicht als studierende Arbeitnehmer oder Familienangehörige von Arbeitnehmern) und die Nichterwerbstätigen, die von Gesetzen zur Sozialversicherung erfasst werden.

Und auch hier sollte der Geltungsbereich erweitert werden. Diese Erweiterung war zum großen Teil wieder durch die Rechtsprechung des Gerichtshofes angeregt.

Hier haben wir zuerst das Urteil VOUGIOUKAS von 1995. Der Gerichtshof hatte anerkannt, dass ,der Gemeinschaftsgesetzgeber (...) jedoch noch nicht die erforderlichen Maßnahmen erlassen [hat], um den sachlichen Geltungsbereich der Verordnung Nr. 1408/71 auf die Sondersysteme für Beamte (...) auszudehnen, so dass (...) eine erhebliche Lücke in der gemeinschaftsrechtlichen Koordinierung der Systeme der sozialen Sicherheit bestehen bleibt ${ }^{\star 35}$.

34 Urteil DE CICCO, vom 14. Dezember 1968, Rs C-19/68, Abs. 12 der Entscheidungsgründe bezüglich der Fragenhintergrundes. 
Somit konnte die Aufrechterhaltung dieser Lücke nicht weiter gerechtfertigt werden, weil der Rat auf diesem Gebiet ,keine Koordinierungsmaßnahme erlassen hat und damit seiner Verpflichtung aus Artikel 51 des Vertrages nicht in vollem Umfang nachgekommen ist" ${ }^{\text {35 }}$.

Dieses Urteil war in gewisser Weise die „Korrektur“ des außergewöhnlich kurzen Urteils LOHMANN vom 8. März 1979, Rs 129/78, wo der Gerichtshof, vorsichtig gesagt, die Möglichkeiten der teleologischen Methode der Auslegung nicht voll genutzt und sich darauf beschränkt hatte, zu erklären, dass der Ausschluss der Sondersysteme der Beamten von der Koordinierung die logische Konsequenz aus Art. 48 Nr. 4 des Vertrages sei, der Beschäftigte im öffentlichen Dienst von der Anwendung der Bestimmungen zur Freizügigkeit der Arbeitnehmer ausschließt. Hierzu auch der Art. 51.

Aber das Urteil VOUGIOUKAS hat ,sicher dazu beigetragen, die Diskussionen im Rat wieder in Gang zu setzen, was letztendlich zur Verabschiedung der Verordnung 1606/98 [vom 22. November 1998] geführt hat “36.

Es war also nicht überraschend, dass in dieser Phase der europäischen Integration die Verordnung 307/99 vom 8. Februar nunmehr die Verordnung 1408/71 auf die Studenten ausdehnte und, dass die große Verordnung Nr. 883/2004 vom 29.4.2004 zu Reform und Vereinfachung schließlich auf die Bürger der Mitgliedstaaten anwendbar ist.

Der Unterschied in der Sichtweise ist, und das sollte man sehen, radikal: während sich die früheren Verordnungen auf die Arbeitnehmer und Selbständigen erstreckten, die gleichzeitig Angehörige der Mitgliedstaaten sind, gilt die neue Verordnung für diese Bürger, egal ob ihre Berufstätigkeit selbständig oder unselbständig ist, also auch für Nichterwerbstätige - das ist auf der Ebene der Koordinierung der Systeme der sozialen Sicherung die Ausstrahlung des Unionsbürgerstatus, der nach Art. 17 des Vertrags die Staatsangehörigkeit eines Mitgliedstaates voraussetzt und ergänzt. Wegen des Gegenstandes der Koordinierungsregelung selbst bleibt nur die schließlich logische Forderung, dass diese Staatsbürger einer Sozialversicherungsgesetzgebung in einem oder mehreren Mitgliedstaaten unterliegen oder unterlagen, was an den Anfang der Rechtsprechung in der Sache UNGER erinnert.

$\mathrm{Zu}$ dieser umfassenden Lösung hat das schon zitierte Urteil SALA beigetragen, das den legal in einem anderen Mitgliedstaat wohnenden Unionsbürgern das Recht zuerkennt, keine Diskriminierung wegen der Staatsangehörigkeit im Verhältnis zu den Staatsangehörigen des Aufnahmestaates im sachlichen Geltungsbereich (wie z.B. die Rechte, die durch die Koordinierungsregelung für die Systeme der sozialen Sicherung garantiert sind) hinnehmen zu müssen.

Es ist schade, dass die Verordnung 883/2004 den Vorschlag für eine Rechtsgrundlage $^{37}$ der Kommission nicht aufrechterhalten hat, das heißt, außer den Artikeln 51 und 235 (heute 42 und 308) auch den Artikel 8a (heute 17) des Vertrags, der unter den mit

35 Urteil VOUGIOUKAS, vom 22. November 1995, Rs 443/93, Rdnr. 31 u. 34.

36 S.V. RAEPENBUSCH, ebenda, S. 42.

37 Amtsblatt C 38 vom 12. Februar 1999, S. 10. 
dem Status der Unionsbürgerschaft verbundenen Rechte das Recht auf Freizügigkeit und freie Wohnsitzwahl auf dem Territorium der Mitgliedsländer vorsieht.

4. Eine Abschlussbemerkung zu den Bürgern von Drittstaaten im Rahmen der Koordinierungsregeln zur Sozialversicherung. Der angeführte Vorschlag der Kommission aus dem Jahre 1999 (Art. 1) zur Einbeziehung aller Versicherten, schloss auch die von einer Sozialversicherungsgesetzgebung eines Mitgliedstaates erfassten Angehörigen von Drittländern mit ein, wenn sie sich in einen anderen Mitgliedstaat begaben.

Dieser Punkt des Vorschlages wurde fallengelassen und der persönliche Geltungsbereich der Verordnung 883/2004 schließt die Angehörigen der Mitgliedstaaten, nicht aber die von Drittländern ein.

Durch die Verordnung 859/2003 sollten die Vorteile der gültigen Koordinierungsregeln mit einigen Anpassungen auf diese ausgedehnt werden, solange sie in einem Mitgliedstaat ihren legalen Wohnsitz haben und sie sich in einer Situation befinden, die über die Grenzen eines einzelnen Mitgliedstaates hinausweist.

Auch hier kam die Rechtsprechung des Gerichtshofes zur Wirkung, sogar in redaktioneller Hinsicht, denn nach den Leitsätzen des Urteils KHALIL aus 2001 können ,die Arbeitnehmer, die als Staatenlose oder Flüchtlinge (..) die von der Verordnung Nr. 1408/71 (...) gewährten Rechte nicht geltend machen, wenn sie sich in einer Situation befinden, die mit keinem Element über die Grenzen dieses Mitgliedstaates hinausweist" 38 .

Es muss noch hervorgehoben werden, dass die Rechtsgrundlage der Verordnung zur Erweiterung (859/2003) nicht die alte Rechtsgrundlage der zu erweiternden Verordnung war (1408/71), sondern es war Art. 63 Nr. 4, als Bestandteil des Titels IV von Teil III des Vertrages zu Visa, Asyl, Einwanderung und anderen, die Freizügigkeit im Personenverkehr betreffenden Politiken, der bezüglich dieser Freiheit nur eine eingeschränkte Reichweite hat.

„Also, verschiedene Bestimmungen des Vertrags für die [Rechtsgrundlage der] Erweiterungsverordnung (Art. 63 Nr. 4), und für die erweiterte Verordnung (hauptsächlich Art. 42), was (...) zukünftig die Ursache von [Auslegungs- und] Anwendungsschwierigkeiten sein kann“39.

38 Urteil KHALIL, vom 11. Oktober 2001, Rs C-95/99, Tenor, Punkt 2.

39 SEBASTIÃO PIZARRO "Quelques notes au sujet de l'observatoire de sécurité sociale pour les travailleurs migrants. Le cas particulier du Règlement d'extension du système communautaire de coordination aux ressortissants des pays tiers" (Anmerkungen zur Beobachtungsstelle über die soziale Sicherheit der Wanderarbeitnehmer. Der Sonderfall der Verordnung zur Erweiterung des Gemeinschaftssystems der Koordinierung auf Drittstaatsangehörige), in Actes du Colloque International "Libre circulation des travailleurs et coordination des régimes de sécurité sociale" (Dokumentation des internationalen Kolloquiums: „Freizügigkeit der Arbeitnehmer und Koordinierung der Systeme der sozialen Sicherheit"), Sakkoulas Publications, Athens-Thessaloniki, 2004, S. 259. Übersetzung des Zitats aus dem Französischen. 
IV. Der Einfluss der Rechtsprechung des Europäischen Gerichtshofes nach dem Urteil DECKER hinsichtlich der Gewährung von Gesundheitsdienstleistungen außerhalb des nationalen Territoriums hängt von der Genehmigung des zuständigen Trägers ab-auf der Suche nach einem fairen Gleichgewicht zwischen den Privatinteressen und dem Allgemeininteresse der nationalen Gesundheitssysteme

1. Art. 22 der Verordnung 1408/71 regelt die Umstände, unter denen Leistungsempfänger, die die Bedingungen der anwendbaren Rechtsvorschriften erfüllen, vom zuständigen Träger die Genehmigung erhalten, sich in einen anderen Mitgliedstaat zur angemessenen medizinischen Behandlung zu begeben. Die Genehmigung für die Gewährung dieser „geplanten“ Gesundheitsdienstleistungen darf jedoch nicht willkürlich verweigert werden, ,wenn die betreffende Behandlung zu den Leistungen gehört, die in den Rechtsvorschriften des Mitgliedstaats vorgesehen sind, in dessen Gebiet der Betreffende wohnt, und wenn er in Anbetracht seines derzeitigen Gesundheitszustands und des voraussichtlichen Verlaufs der Krankheit diese Behandlung nicht in einem Zeitraum erhalten kann, der ... normalerweise erforderlich ist" (Art. 22, Absatz 2, Abschnitt 2 der Verordnung).

Diese Genehmigung für eine angemessene medizinische Behandlung (Produkte und Krankenversorgung) in einem anderen als dem zuständigen Staat sollte also nach dem Urteil DECKER Gegenstand der Rechtsprechung des Gerichtshofes werden - nicht mehr allein im Lichte der Auslegung des genannten Artikels 22 im Hinblick auf die Freizügigkeit im Personenverkehr, sondern im Sinne der Vereinbarkeit der nationalen Gesetzgebung, die eine solche Genehmigung fordert, mit den Bestimmungen des EGVertrages in Bezug auf den freien Waren- und vor allem Dienstleistungsverkehr.

Mit diesem Urteil begann ein neue, außerordentlich vielfältige Periode, die sich unter anderem mit den Urteilen KOHLL aus dem gleichen Jahr, VANBRAEKEL aus 2001, SMITS und PEERBOMS ebenfalls aus 2001, MULLER-FAURÉ und PATRICIA INIZAN, jeweils aus 2003 und WATTS aus 2006 fortsetzte.

Besonders interessant ist, dass es in den ersten beiden Rechtssachen direkt um die Frage der Verweigerung der Rückerstattung für außerhalb des Territoriums erbrachte medizinische Leistungen ging und daraufhin besonders in den Folgeurteilen die wirklich zentrale Frage der Genehmigung analysiert wurde, und zwar durch die Prüfung der Vereinbarkeit der nationalen Gesundheitssysteme mit den entsprechenden Artikeln des EG-Vertrages.

2. Ohne die Gültigkeit von Artikel 22 in Frage zu stellen erklärte der EuGH, dass „der Umstand, dass eine nationale Maßnahme (die Notwendigkeit der Genehmigung) möglicherweise einer Bestimmung des abgeleiteten Rechts - hier dem Art. 22 der Ver- 
ordnung Nr. 1408/71 - entspricht, ... nicht zur Folge [hat], dass sie nicht an den Bestimmungen des EG-Vertrages zu messen wäre“; hingegen regelt dieser Artikel „bei zweckgerichteter Auslegung ... nicht den Fall (und hindert daher keinesfalls daran), dass die Kosten für eine in einem anderen Mitgliedstaat ohne vorherige Genehmigung erbrachte Behandlung zu den Sätzen erstattet werden, die im Versicherungsstaat gelten. “40

Eine solche durch den zuständigen Träger direkt an den Versicherten nach den Sätzen des Versicherungsstaates gezahlte Rückerstattung hat nichts zu tun mit der durch diesen Träger geleisteten Erstattung an den Träger des Staates, der die Leistung erbracht hat, die auf der Grundlage der in diesem Staat geltenden Sätze nach Maßgabe des anzuwendenden Erstattungssystems gem. Art. 22 berechnet wird.

Bei der Prüfung der Vereinbarkeit der nationalen Gesetzgebung mit dem Gemeinschaftsrecht entschied der EuGH im Urteil KOHLL, dass „eine nationale Regelung, die die Erstattung der Kosten für Zahnbehandlung durch einen Zahnarzt in einem anderen Mitgliedstaat nach den Tarifen des Versicherungsstaats von der Genehmigung des Trägers der sozialen Sicherheit des Versicherten abhängig macht, ... gegen die Artikel 59 und 60 EG-Vertrag [verstößst]. ${ }^{41}$ (Gleiche Überlegungen wurden im Urteil DECKER wegen der Rückerstattung der Kosten für eine Brille dargelegt).

Trotz dieser grundsätzlichen Ablehnung des Genehmigungssystems aufgrund seiner Unvereinbarkeit mit dem EG-Vertrag schließt der Gerichtshof gleichzeitig drei zwingende Gründe des Allgemeininteresses nicht aus, die Beschränkungen gegenüber den genannten Vertragsbestimmungen rechtfertigen könnten:

das Vorliegen einer „erheblichen Gefährdung des finanziellen Gleichgewichts des Systems der sozialen Sicherheit"

die „Aufrechterhaltung einer ausgewogenen, allen zugänglichen ärztlichen und klinischen Versorgung“"

die „Erhaltung eines bestimmten Umfangs der medizinischen und pflegerischen Versorgung oder eines bestimmten Niveaus der Heilkunde im Inland, die für die Gesundheit oder selbst das Überleben [der] Bevölkerung" 42 als erforderlich angesehen wird.

Diese zwingenden Gründe dienen auch heute als Orientierung und wurden in der nachfolgenden Rechtsprechung weiterentwickelt, sowohl in Bezug auf ambulante Leistungen wie in den Rechtssachen DECKER und KOHLL (in denen sich der Gerichtshof im Prinzip für die Nichtvereinbarkeit der Genehmigung mit dem Vertrag entschied), als auch in Bezug auf die stationären Leistungen, die hauptsächlich in den späteren Rechtssachen zur Entscheidung anstanden (bei denen sich der Gerichtshof im Prinzip für die Vereinbarkeit mit dem Genehmigungssystem aussprach).

40 Urteil DECKER, ebenda, Rdnr. 27 und 29.

41 Urteil KOHLL vom 22. April 1998, Rs C-158/96, Tenor.

42 Urteil KOHLL, ebenda, Rdnr. 41, 50 und 51. 
Interessant ist auch der Tenor des Urteils VANBRAEKEL, da es dem Versicherten gemäß Artikel 59 (heute 49) EG-Vertrag das Recht auf eine ergänzende Erstattung für die Krankenhausdienstleistungen zugesteht, wenn die Erstattung von Kosten in einem Aufenthaltsmitgliedstaat ,niedriger als diejenige ist, die sich aus der Anwendung der im Mitgliedstaat der Versicherungszugehörigkeit geltenden Rechtsvorschriften im Fall einer Krankenhauspflege in diesem Staat ergeben würde..." “43.

Der Einfluss dieser Rechtsprechung auf die nationalen Gesundheitssysteme mit dem Ziel, sie mit dem freien Waren- und Dienstleistungsverkehr vereinbar zu machen, kann zur Anpassung der Koordinierungsregelung führen, die von nicht geringerem Einfluss ist.

Nun ist es im Rahmen dieses Vortrags unmöglich, auf alle Aspekte und Auswirkungen einzugehen, und deshalb soll versucht werden, dem Leitfaden der Grundwesenszüge durch die Wiedergabe einiger diesbezüglicher Urteilssprüche und Erwägungen zu folgen, wobei vor allem das Urteil WATTS als Bezugspunkt dienen soll, das die vorhergehende Rechtsprechung konsolidiert und ergänzt.

3. Indem er bekräftigt, dass „,die Anwendbarkeit von Artikel 22 der Verordnung Nr. 1408/71 (...) nicht ausschließt, dass der Betroffene parallel dazu gemäß Art. 49 EG einen Anspruch auf Zugang zu Leistungen der Gesundheitspflege in einem anderen Mitgliedstaat unter Bedingungen der Kostenübernahme hat, die sich von denen [des genannten Artikels] unterscheiden“44, verleiht der EuGH der Öffnung eines zweiten Weges für den Zugang zu diesen Leistungen außerhalb des nationalen Territoriums Nachdruck, nunmehr direkt gestützt auf den EG-Vertrag, über den in der Verordnung enthaltenen traditionellen Weg hinaus.

In Bezug auf die Rechtsprechung nach den Urteilen DECKER und KOHLL und ausgehend von der Unterscheidung zwischen der Versorgung in einem Krankenhaus und außerhalb eines Krankenhauses lässt sich demnach feststellen, dass die Artikel des Vertrages zu den genannten wirtschaftlichen Freiheiten ,sowohl nicht einer Regelung eines Mitgliedstaates entgegenstehen, die die Übernahme der Kosten für die Versorgung in einer Krankenanstalt in einem anderen Mitgliedstaat davon abhängig macht, dass ... eine vorherige Genehmigung erteilt wird“45, wie andererseits die gleichen Artikel diesen Rechtsvorschriften „entgegenstehen“, wenn sie die Übernahme der Kosten für die Versorgung in einem anderen Mitgliedstaat außerhalb eines Krankenhauses ... davon abhängig machen, dass ... eine vorherige Genehmigung erteilt wird ${ }^{46}$.

Über die typischen Dienstleistungen in einem Krankenhaus hinaus, die im Verständnis des EuGH ,im Vergleich zu den medizinischen Leistungen, die die frei praktizie-

43 Urteil VANBRAEKEL vom 12. Juli 2001, Rs C-368/98, Tenor.

44 Urteil WATTS vom 16. Mai 2006, Rs C-372/04, Rdnr. 48.

45 Urteil SMITS und PEERBOOMS vom 12. Juli 2001, Rs C-157/79, Tenor.

Vgl. auch Urteil MULLER-FAURÉ, ebenda, Tenor (erster Teil), Urteil INIZAN, Rs C-56/01 vom 23. Oktober 2003, Tenor und Urteil WATTS, ebenda, Punkt 2 im Tenor.

46

Urteil MULLER-FAURÉ, ebenda, Tenor (zweiter Teil). 
renden Ärzte in ihrer Praxis ... erbringen, unbestreitbar Besonderheiten aufweisen“47, geht es bei allem darum - sei es im Fall der Nichtvereinbarkeit der nationalen Regelungen mit den Bestimmungen des EG-Vertrages (Leistungen außerhalb des Krankenhauses), sei es im Fall der Vereinbarkeit zwischen diesen Rechtsvorschriften (Leistungen in einer Krankenanstalt) - ein Gleichgewicht zwischen den privaten Interessen der Leistungsempfänger und dem Allgemeininteresse der Gesundheitssysteme zu erreichen.

Hinsichtlich der erstgenannten Leistungen muss das Verbot der Genehmigung unbeschadet der Ausnahmen funktionieren, die gegebenenfalls die Notwendigkeit der Genehmigung rechtfertigen, insbesondere wenn die oben genannten zwingenden Gründe im Allgemeininteresse vorliegen; hinsichtlich der Versorgung in einer Krankenanstalt verhindert die Anerkennung der Genehmigungspflicht in der Regel nicht, dass die Genehmigung gegeben wird, wobei eine eventuelle Ablehnung objektiv begründet sein muss.

„Ein System der vorherigen Genehmigung ist daher jedenfalls nur dann trotz des Eingriffs in eine solche Grundfreiheit gerechtfertigt, wenn es auf objektiven und nicht diskriminierenden Kriterien beruht, die im Voraus bekannt sind...“48.

4. Konkret gesagt ist der Gerichtshof der Auffassung, dass „den nationalen Stellen, die ... zuständig sind, nicht das Recht abgesprochen werden [kann], ... ein System von Wartelisten einzuführen, das darauf abzielt, die Erbringung der Behandlungen zu planen und Prioritäten nach Maßgabe der verfügbaren Mittel und Kapazitäten festzulegen““49

Die Genehmigung kann nur dann unter Berufung auf das Bestehen einer Wartezeit versagt werden, wenn unter den Umständen des konkreten Falls nicht der zeitliche Rahmen überschritten wird, ,der unter Berücksichtigung einer objektiven medizinischen Beurteilung des klinischen Bedarfs des Betroffenen im Hinblick auf seinen Gesundheitszustand, seine Vorgeschichte, die voraussichtliche Entwicklung seiner Krank-

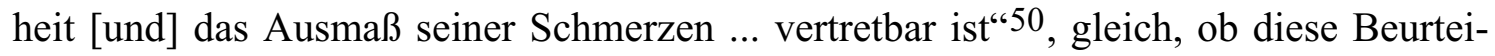
lung im Moment des Genehmigungsantrags vorgenommen wurde oder später, „falls sich der Gesundheitszustand verschlechtert." 51

Finanzielle Gründe (höhere Ausgaben für die Krankenhausbehandlung als die Kosten einer gleichartigen Behandlung im Versicherungsstaat) können „kein legitimer Grund für die Versagung der Genehmigung sein“. 52

Im Gegensatz dazu ist die Versagung begründet, wenn „,die gleiche oder eine für den Patienten ebenso wirksame Behandlung rechtzeitig in dessen Wohnmitgliedstaat erlangt werden kann". 53

47 Urteil SMITS und PEERBOOMS, ebenda, Rdnr. 76.

48 Urteil WATTS, ebenda, Rdnr. 116,67,68,69,73,61,77,106,139,144,145 und 147.

49 S. Fn. 48.

50 S. Fn. 48.

51 S. Fn. 48.

52 S. Fn. 48. 
Die Versagung ist auch dann begründet, wenn als Grund für die Erlangung einer Krankenhausbehandlung in einem anderen Mitgliedstaat das Argument vorgebracht wird, sie ,ungeachtet von Erwägungen der medizinischen Notwendigkeit ... zu einem früheren Zeitpunkt ... zu erhalten". 54

Aber selbst wenn die Beschränkung der Freizügigkeit im Dienstleistungsverkehr tatsächlich mit derartigen zwingenden Gründen gerechtfertigt werden kann, muss sichergestellt werden, dass die Beschränkung „,nicht über dasjenige hinausgeht, was zu diesem Zweck objektiv notwendig ist, und dass das gleiche Ergebnis nicht durch weniger einschneidende Regelungen erreicht werden kann" 55 - nach dem Grundsatz der Verhältnismäßigkeit.

Hinsichtlich der zusätzlichen, nicht als Sachleistungen im Sinne von Art. 22 der Verordnung geltenden Ausgaben für Reise- und Unterbringungskosten für den Fall, dass dem Patienten genehmigt wurde, sich zur Krankenhausbehandlung in einen anderen Staat zu begeben, war der Gerichtshof der Auffassung, dass „die Rechtsvorschriften eines Mitgliedstaates gegen Art. 49 EG verstoßen, wenn sie die Übernahme [dieser] Nebenkosten ausschließen, ... während sie die Übernahme dieser Kosten vorsehen, wenn die Behandlung in einer Einrichtung des fraglichen nationalen Dienstes erfolgt" ${ }^{* 56}$.

Ein letztes wichtiges Problem im Hinblick auf die Verteilung der Zuständigkeiten zwischen dem nationalen Recht und dem Gemeinschaftsrecht wurde dem Gerichtshof vorgelegt und besteht im Grunde genommen in der Frage, ob die sich aus dieser Rechtsprechung gemäß Art. 49 EG-Vertrag und Art. 22 der Verordnung ergebende Forderung so zu verstehen ist, dass sie den Mitgliedstaaten die Verpflichtung auferlegt, „Krankenhausbehandlungen in anderen Mitgliedstaaten ohne Rücksicht auf Haushaltszwänge zu finanzieren, und wenn ja, ob eine solche Verpflichtung mit Art. 152 Absatz 5 EG vereinbar ist" ${ }^{\text {57 }}$ (diese Bestimmung berührt die den Mitgliedstaaten vorbehaltene Zuständigkeit für die soziale Sicherheit im Hinblick auf die Bereitstellung von Gesundheitsdienstleistungen und ärztlicher Betreuung).

Die Begründung der Antwort ist ein Eckpfeiler und ein Schlüssel für das Verständnis der Rechtsprechung des EuGH auf diesem Gebiet: „die sich [aus diesen Artikeln] ergebenden Anforderungen ... [entspringen] vielmehr dem Bemühen um ein Gleichgewicht zwischen dem Ziel der Freizügigkeit der Patienten einerseits und den nationalen Zwängen der Planung der verfügbaren Krankenhauskapazitäten, der Beherrschung der Gesundheitskosten und des finanziellen Gleichgewichts der Systeme der sozialen Sicherheit andererseits" 58 . 
Nach der Darlegung, dass diese Anforderungen nicht gegen Art. 152 Abs. 5 verstoßen, bestätigte der Gerichtshof, dass ,diese Bestimmung jedoch nicht ausschließt, dass die Mitgliedstaaten nach anderen Vertragsbestimmungen wie Art. 49 ... verpflichtet sind, Anpassungen in ihren nationalen Systemen der sozialen Sicherheit vorzunehmen, ohne dass dies als Eingriff in ihre souveräne Zuständigkeit in dem betreffenden Bereich angesehen werden könnte. “59

5. Wie ist mit dieser Rechtsprechung umzugehen? Welche Auswirkungen hat sie? Wie kann die Übereinstimmung der Rechtsvorschriften der Mitgliedstaaten im Gesundheitswesen mit den wesentlichen wirtschaftlichen Freiheiten im EG-Vertrag sichergestellt werden? Eine solche Übereinstimmung muss im Übrigen nicht nur dann eintreten, wenn eine Unvereinbarkeit erklärt wurde, sondern auch, wenn der EuGH die Vereinbarkeit zwischen beiden zwar anerkennt, sie aber Bedingungen und Kriterien unterwirft, die die Ermessensbefugnis der nationalen Behörden berücksichtigen sollen.

Eine Veränderung oder Anpassung dieser Rechtsvorschriften bzw. ihre Koordinierung ist unvermeidbar, unabhängig davon, wie die Gesundheitsdienste organisiert oder gestaltet sind. Sollte das durch eine Richtlinie oder eine Verordnung geschehen? Der erste Weg gäbe den Mitgliedstaaten mehr Handlungsspielraum, da er ihnen die Zuständigkeit für die Form und die Mittel überlässt, aber er könnte keine vollständige Garantie für das grundlegende Ziel geben, eine ,einheitliche Umsetzung in nationales Recht“ zu sichern, die nur durch eine ebenfalls einheitliche „Auslegung“ der Rechtsprechung gewahrt wäre.

Daher scheint es ratsam zu sein, sich für eine Verordnung zu entscheiden, nicht nur, weil sie in all ihren Elementen verbindlich und direkt anwendbar wäre und in gewisser Weise die genannte Unabwägbarkeit abmildern würde, sondern auch, weil sie es gestattet, die lange und reiche Erfahrung der funktionierenden Koordinierung der Systeme der sozialen Sicherheit zu nutzen, zu denen die Gesundheitsdienstleistungen gehören. Es ist richtig, dass dieses System die Freizügigkeit im Personenverkehr zur Hauptgrundlage hatte, aber die Lehren aus seiner praktischen Anwendung sind übertragbar auf die anderen Freiheiten.

Der Punkt ist, dass die „Koordinierung der Rechtsprechung“ alle - und zwar alle fraglichen Interessen berücksichtigen muss, was der EuGH einmal mehr im Urteil WATTS unterstrichen hat.

Zur Veranschaulichung eines dieser Interessen, nämlich die ständige Erhaltung der Behandlungskapazität bzw. der medizinischen Kompetenz auf nationalem Territorium, das als wesentlich für die öffentliche Gesundheit und das Überleben der Bevölkerung anzusehen ist, sei eine extrem hypothetische Abfolge aufgestellt:

a) massenhafte Nachfrage der Leistungsempfänger von Mitgliedstaat A nach Gesundheitsleistungen einer hoch spezialisierten Einrichtung in Mitgliedstaat B;

59 S. Fn. 48. 
b) Aufgabe der Tätigkeit in einer ähnlichen Einrichtung in Mitgliedstaat A, weil sie nicht mehr genutzt wird und weil deswegen Fixkosten für Personal und Ausrüstungen eingespart werden müssen;

c) Ausbruch einer breiten Epidemie mit Breitenwirkung in den Staaten A und B;

d) in der Folge völlige Auslastung der spezialisierten Einrichtung hauptsächlich durch die Versorgung der Einwohner von Staat B (selbst wenn ein gleicher Zugang zu den Gesundheitsleistungen gewährt worden ist) und

e) demzufolge unzureichende Versorgung der Bürger von Staat A ... .

Zusammengefasst: Die Gewährung von Gesundheitsleistungen in einem anderen als dem eigenen Land darf nicht als absolute Folge dreier grundlegender Freiheiten (Freizügigkeit im Personen-, Waren- und Dienstleistungsverkehr) verstanden werden, noch viel weniger, wenn dieses Verständnis im Sinne eines uneingeschränkten Rechts der Versicherten auf freie Auswahl des Gesundheitssystems eines Mitgliedstaates zu begreifen wäre.

Auch hierbei erklärte der Gerichtshof mit außerordentlicher Klarheit, dass nach einem Gleichgewicht zwischen der Durchsetzung der Privatinteressen, die die Patienten begreiflicherweise vom diesbezüglichen Freizügigkeitsrecht erwarten, und den Erfordernissen des Allgemeininteresses der nationalen Gesundheitssysteme gesucht werden muss, also letztendlich ein Ausgleich zu schaffen ist zwischen den mit den Prinzipien und der Wirtschaftlichkeit dieser Systeme verbundenen Interessen einerseits und den sich aus den Freizügigkeitsrechten der Gemeinschaft ergebenden Anforderungen auf der anderen Seite.

\section{Die Rechtsprechung des Europäischen Gerichtshofes und die künftige Anwendung der Verordnung Nr. 883/2004}

1. Es sei eine zweite persönliche Anmerkung gestattet: Ich hatte das Glück, als Vertreter Portugals einen Beitrag zur fachlichen Ausarbeitung der Verordnung Nr. 883/2004 leisten zu dürfen, für die von 1999 bis 2004 verhandelt wurde, und in meiner Erinnerung waren die schwierigsten Verhandlungsphasen die besten, als die Suche nach Lösungen auf dem Spiel stand, die ein ,faires Gleichgewicht“ erforderten und als Wege zum Kompromiss und Konsens dringend erforderlich waren.

Abgesehen davon, dass man zweifellos hätte weiter gehen können - ein solches Bestreben ist immer möglich - hat die neue Koordinierungsregelung das Verdienst, durchweg vereinfacht zu sein und außerdem unleugbare Verbesserungen und echte Reformen festzuschreiben, die das Bemühen um den Schutz der europäischen Bürger zum Ausdruck bringen, die einen Ortswechsel innerhalb der Gemeinschaft vollziehen oder vollzogen haben. 
Dazu ist es ausreichend, die Erweiterung des persönlichen und sachlichen Anwendungsbereiches der Verordnung und die unterschiedslose Ausweitung des allgemeinen Systems der Familienleistungen auf Arbeitnehmer, Rentner und Personen, die Waisenkinder in ihrer Obhut haben, zu nennen.

In diesem Zusammenhang sei daran erinnert, dass die neue Verordnung schon in Kraft ist, aber ihre Anwendung hängt vom Inkrafttreten der noch im Prozess der Ausarbeitung befindlichen Durchführungsverordnung ab.

Da sie aus Sicht der Bereicherung der teleologischen Auslegungsmethode interessant erscheinen, sollen hier beispielhaft einige innovative Lösungen genannt werden, sowohl in Bezug auf die drei Anwendungsgebiete als auch auf die drei Grundprinzipien der Koordinierungsregelung. An erster Stelle ist die Bedeutung der 45 Erwägungen dieser Verordnung hervorzuheben, wobei auch die Auslegung ihrer Regeln Berücksichtigung finden muss.

Danach werden aus einer sozusagen umgedrehten Perspektive hinsichtlich der künftigen Rechtsprechung zwei Bestimmungen ausgewählt, die Zweifel bei der Auslegung im Hinblick auf die praktische Anwendung hervorrufen könnten und bei denen ein Eingreifen des Gerichtshofs auf der Grundlage von Art. 234 EG-Vertrag nicht ausgeschlossen wäre, damit es zu einer einheitlichen Auslegung kommt.

2. Acht Erwägungen in der Verordnung Nr. 883/200460 haben einen direkten Bezug zum EuGH (indirekt sind es jedoch mehr, wenn man bedenkt, dass die Textfassung anderer Erwägungen seine Rechtsprechung aufnimmt).

Unter diesen acht sind zu erwähnen die Erwägung Nr. 31, derzufolge es ,nach Auffassung des Gerichtshofs Sache des nationalen Gesetzgebers ist, derartige Rechtsvorschriften (gegen das Zusammentreffen von Rechtsansprüchen) zu erlassen, wobei der Gemeinschaftsgesetzgeber die Grenzen festlegt, in denen die nationalen Kürzungs-, Ruhens- und Entziehungsvorschriften anzuwenden sind“", und die Nr. 37, wo bekräftigt wird, dass „der Gerichtshof wiederholt festgestellt hat, dass Vorschriften, mit denen vom Grundsatz der Exportierbarkeit der Leistungen der sozialen Sicherheit abgewichen wird, eng ausgelegt werden müssen. Dies bedeutet, dass sie nur auf Leistungen angewendet werden können, die den genau festgelegten Bedingungen entsprechen...“.

Daher ist davon auszugehen, dass die vom Gerichtshof verwendete teleologische Auslegungsmethode in den Erwägungen der Verordnung Nr. 883/2004 eine zusätzliche Argumentationsgrundlage findet, insbesondere im Hinblick auf die Auslegung einiger ihrer innovativen Bestimmungen.

Beim Anwendungsbereich sind folgende Neuerungen hervorzuheben:

die Anwendung der neuen Verordnung auf die Bürger der Mitgliedstaaten geht über den Personenkreis der Arbeitnehmer (zu denen die Beamten gehören) und

60 Das sind die Erwägungen 3, 9, 21, 24, 30, 31, 34 und 37. 
Studenten hinaus und umfasst nunmehr nicht erwerbstätige Personen (Art. 1), was wie gesagt dem Status als Unionsbürger entspricht;

durch die Verordnung 859/2003 wurde die Koordinierungsregelung der Verordnung Nr. 1408/71 auch auf die Bürger von Drittländern ausgeweitet;

- auch wenn keine so genannte Beispielliste der Sozialversicherungsgebiete aufgenommen worden ist, die im Prinzip den sachlichen Geltungsbereich mit allen Rechtsvorschriften zur sozialen Sicherheit in Übereinstimmung gebracht hätte, was eher im Einklang mit Art. 42 EG-Vertrag wäre, war es möglich, diesen Geltungsbereich auf die Vorruhestandsleistungen auszudehnen (Art. 66, der in Verbindung mit den Allgemeinen Bestimmungen und der Erwägung Nr. 33 gesehen werden muss);

- gewissermaßen im Zusammenhang mit dem territorialen Rahmen ist die Gleichbehandlung61 nicht mehr vom Wohnaufenthalt der Bürger auf dem Gebiet eines Mitgliedstaates abhängig (Art. 4).

Bei den Grundprinzipien gibt es die folgenden Neuerungen:

- das Gleichbehandlungsprinzip 62 verbietet nunmehr durch eine allgemeine, nicht mehr fallbezogene Bestimmung ${ }^{63}$ die indirekte Diskriminierung durch die Nichtgleichstellung von Leistungen, Einkünften und Sachverhalten auf dem Gebiet der Mitgliedstaaten und sichert auf diesem Wege die Gleichwertigkeit der Rechtswirkungen im Sinne der Gesetzgebung des zuständigen Staates in Bezug auf Ereignisse, die in einem anderen Mitgliedstaat eingetreten sind (Art. 5);

das Prinzip der Ausschließlichkeit der anzuwendenden Gesetzgebung wurde nun in Einklang gebracht mit dem Begriff als solchem, d.h. dass eine Person, auf die die Verordnung Anwendung findet, den Rechtsvorschriften nur eines Mitgliedstaates unterliegt und nicht mehr der Gesetzgebung von mehr als einem Mitgliedstaat unterworfen ist (Art. 11);

das Prinzip der Wahrung der Rechte im Hinblick auf erworbene Ansprüche bekommt mehr Gewicht durch die Zusammenrechnung der Zeiten, nicht nur durch die Erweiterung ihrer Anrechenbarkeit, sondern auch durch die Berücksichtigung in einer allgemeinen Bestimmung (Art. 6); gleichfalls ist nun das Grundprinzip der Wahrung erworbener Ansprüche im Einklang mit Art. 42 EG-Vertrag in dem

61 Anzumerken ist die Angleichung zwischen Art. 4 zur Gleichbehandlung und Art. 17 EG-Vertrag zur Unionsbürgerschaft im Hinblick auf die Reihenfolge, in der die Rechte und Pflichten aufgeführt sind (in früheren Verordnungen war die Reihenfolge umgekehrt - Pflichten und Rechte).

62 In der portugiesischen Fassung von Art. 5 der Verordnung Nr. 883/2004 wurde für den Begriff, ,assimilação“ - Gleichstellung (der Sachverhalte) auch, was bedeutsam ist, der Ausdruck ,igualdade de tratamento" - Gleichbehandlung (der Sachverhalte) verwendet.

63 Diese Bestimmung ist ein bezeichnendes Beispiel für die Bedeutung der Erwägungen bei der Auslegung der neuen Verordnung. Nicht weniger als vier Erwägungsgründe (9 bis 12) sind dem Gleichstellungsgrundsatz gewidmet, der „explizit aufgenommen und ausgeformt werden sollte, wobei Inhalt und Geist der Gerichtsentscheidungen zu beachten sind", der aber von vielleicht übertriebenen Vorbehalten umgeben ist, die verhindern sollen, daß seine Anwendung ,nicht zu sachlich nicht zu rechtfertigenden Ergebnissen führt" (Erwägungen 9 und 12)“. 
Maße, dass die Aufhebung der Wohnortsklauseln ebenso als allgemeine Bestimmung auf alle Geldleistungen im Sinne der Rechtsvorschriften eines oder mehrerer Mitgliedstaaten bzw. der Verordnung und nicht mehr nur auf die zurückgestellten Leistungen anzuwenden ist (Art. 7).

3. Die beiden für umstrittene Auslegungen anfälligen Bestimmungen ${ }^{64}$ beziehen sich zum einen auf die Geldleistungen bei Arbeitslosigkeit und zum anderen auf die unterschiedliche Ergänzung der Familienleistungen. Konkret gesagt:

die Möglichkeit der Verlängerung des aktuellen Zeitraums für die Gewährung von Leistungsansprüchen beim Aufenthalt Arbeitsloser außerhalb ihres Staates um höchstens 3 Monate (Art. 64 letzter Absatz); und

- die nicht notwendige Gewährung eines Unterschiedsbetrags bei den Familienleistungen bei der Zusammenrechnung von Ansprüchen, wenn der entsprechende Leistungsanspruch ausschließlich durch das Kriterium Wohnort ausgelöst wird (Art. 68, Abs. 2, letzter Satz).

Die erste Bestimmung bezieht sich auf die vom Gemeinschaftsgesetzgeber gestärkte Möglichkeit der Zahlung von Geldleistungen an Arbeitslose, die sich außerhalb ihres Landes aufhalten, indem eine Verlängerung um einen Zeitraum von höchstens drei Monaten zugelassen wird. Zwar handelt es sich um eine Verbesserung, aber wenn keine Kriterien für eine Verlängerung vorliegen, besteht das Risiko, dass dem zuständigen Träger ein gewisser willkürlicher Spielraum bleibt, was Zweifel hervorrufen kann, insbesondere wenn die Ausübung der Freizügigkeit im Personenverkehr und das Gleichbehandlungsprinzip herangezogen werden.

Die zweite Bestimmung, die ebenfalls im Kontext der Verbesserung der Koordinierungsregelung zu sehen ist - die Einbeziehung nicht erwerbstätiger Personen - ändert die Regeln für die Gewährung des Unterschiedsbetrags bei Familienleistungen und unterscheidet, ob der Anspruch darauf in Abhängigkeit vom Wohnort oder infolge einer Erwerbstätigkeit erworben oder durch den Bezug einer Rente ausgelöst wurde. Im ersten Fall kann der Betrag möglicherweise nicht zugebilligt werden, in den anderen beiden Fällen muss er gewährt werden.

Auch hierbei wird die Frage wichtig sein, ob ein solcher Unterschied durch objektive Gründe gerechtfertigt und angemessen ist, wobei dafür eine Entscheidung des Gerichtshofs notwendig sein kann.

Es kann durchaus sein, dass die Rechtsauslegung dieser Bestimmungen keine neuen qualitativen Probleme hervorruft. Die Rechtsprechung des Gerichtshofs ist ja grundsätzlich nicht unbedingt durch die Änderung der Koordinierungsregelung berührt, denn wie zu sehen war haben sich viele der auf der teleologischen Methode beruhenden Ur-

64 Es steht eindeutig nicht die Rechtschaffenheit der vorgesehenen Lösungen in Frage, denn sie sind wie im Text dargestellt wichtige Entwicklungen der Koordinierungsregelung. Es handelt sich allerdings um mögliche Auslegungsprobleme, die sich bei der konkreten Anwendung ergeben könnten. 
teile aus der Auslegung der heute noch gültigen und aktuellen Verordnung Nr. 3/1958 ergeben.

Das ist so, weil die fortdauernde Umsetzung der im EG-Vertrag verankerten Freiheiten, insbesondere der in Art. 42 als Ziel formulierten Freizügigkeit im Personenverkehr die Auslegung nicht nur dieser Bestimmung, sondern der für ihre Anwendung angenommenen Verordnungen bedingt.

Art. 42 ist mit Ausnahme des Entscheidungsverfahrens, das nunmehr eine gemeinsame Entscheidung von Europäischem Parlament und Rat vorsieht, ohne substanzielle Änderungen in seiner ursprünglichen Fassung erhalten geblieben. 65

Damit soll gesagt werden, dass die Entscheidungen eventueller Vorabentscheidungsverfahren aller Wahrscheinlichkeit nach nicht überraschend sein werden.

4. Ohne in die ,alte Diskussion“ über die Wirksamkeit der Entscheidungen eintreten zu wollen (die auf den strittigen Fall als Präzedenzfall oder rechtskräftiges Urteil begrenzt ist) kann man sagen, dass die Rechtsprechung des EuGH auf dem Gebiet der Koordinierung der Systeme der sozialen Sicherheit die Autorität und den Mehrwert besitzt, sich begründet (vielleicht begründeter als auf anderen Gebieten) einer interpretierten Vorschrift anzuschließen, die dann eigenständig so angeführt wird, wie sie vom Gerichtshof ausgelegt wird.

Diese Rechtsprechung wird von den nationalen Gerichten und, wie könnte es anders sein, auch von den Regierungen befolgt, sowohl von den Regierungen als solchen wie auch in der Form der EU-Institution (Ministerrat). Das geht soweit, dass sie oft berücksichtigt wird, wenn eine Aktualisierung oder Reform der Verordnungen ansteht - in einer Art dialektischer Interaktion, in dem Vorschriften angesichts entstandener Zweifel bei ihrer Anwendung ausgelegt und recht häufig dann auch ,aktualisiert“ werden, indem der Tenor der Rechtsprechung aufgegriffen wird.

Und was ist im Hinblick auf den Gerichtshof zu Kraft und Wirkung dieser Rechtsprechung zu sagen? „Regierung der Richter“ oder ganz einfach „Wächter über das Recht in der Auslegung und Anwendung" des EG-Vertrages? Die Antwort auf diese Frage birgt in sich selbst schon eine ungerechte Kritik und enthält zwei Elemente, eines von allgemeiner Bedeutung und ein zweites von spezifischem Belang.

65 Er bleibt auch im Wesentlichen im Vertrag erhalten, der eine Verfassung für Europa begründet (Art. III-136), erneut mit Ausnahme des Entscheidungsverfahrens, wofür nunmehr eine qualifizierte Mehrheit vorgeschlagen wird und das Eingreifen des Europäischen Rates erforderlich sein kann. In diesem Zusammenhang ist, neben dem Art. III-136, dem heutigen Art. 42, auch Art. III-125, Absatz 2 zu nennen, in dem im Gegensatz zum geltenden Vertrag die Möglichkeit eingeräumt wird, dass der Rat einstimmig Maßnahmen, die die soziale Sicherheit oder den sozialen Schutz betreffen, beschließen kann,. Das Ziel dieser Bestimmung, einerseits "die Ausübung des Rechts aller Unionsbürger, sich frei zu bewegen und aufzuhalten, zu erleichtern" und andererseits die systematische Anerkennung ihres Anspruchs auf die als Titel formulierte "Nichtdiskriminierung und Bürgerschaft" kann hier als zusätzliche Bekräftigung von Art. III-136 gelten. Diesem Ziel und dieser Anerkennung wird der Gerichtshof stets seine besondere Aufmerksamkeit schenken, wenn ein neuer Vertrag in Kraft tritt. 
Das erste hängt mit der Mission zusammen, die Einheitlichkeit und Kohärenz der Auslegung bei der Anwendung des Gemeinschaftsrechts durch alle Mitgliedstaaten zu garantieren, weil das eine wesentliche Bedingung und Teil der Idee und Funktionsweise der Gemeinschaft ist.

Das zweite Element ergänzt das erste auf einem Gebiet mit 27 einzelstaatlichen Systemen, wo Nichtdiskriminierung und Solidarität als Prüfstein dienen müssen. Es hat zu sichern, dass den Menschen, die die im EG-Vertrag verankerten Grundfreiheiten nutzen, nicht ihre Rechte der sozialen Sicherheit beschnitten werden oder sie sie sogar verlieren, nur weil eine Vorschrift von den mitgliedstaatlichen Institutionen unterschiedlich ausgelegt werden kann.

Das erklärt, dass sowohl die ,alte Kritik“ am Eindringen in die Zuständigkeiten der eigentlichen Rechtsprechungsorgane als auch die alte Diskussion über die Verbindlichkeit ihrer Entscheidungen nichts anderes als die Folge der dem Gerichtshof eigenen, ihm übertragenen Funktionen ist, insbesondere wenn sie im gemeinsamen europäischen Rahmen von Art. 234 ausgeübt werden. Und hier sei am Ende wieder der Anfang aufgegriffen - es wäre tatsächlich absurd, wenn es diesen Artikel nicht gäbe. 


\section{Diskussionsbericht zu Teil III}

\section{Diskussionsleitung: Matthias von WULFFEN}

Nach dem Koreferat von Pizzarro entbrannte eine lebhafte Diskussion über die Rechtsprechung des EuGH zum Eltern- und Erziehungsgeld. Diese sei eines der Musterbeispiele dafür, dass der EuGH in seiner Zielsetzung, die Wanderarbeitnehmer zu schützen, über das Ziel hinausgeschossen sei und daher nun zurückrudere. Dies zeige sich bei Gegenüberstellung der Rechtssachen Höver/Zachow und Dodl/Oberhollenzer. Zunächst einmal liege der EuGH falsch, wenn er in der Entscheidung Höver/Zachow feststelle, dass die Elterngeldberechtigung eine abgeleitete Sicherung im Sinne von Art. 73 VO 1408/71 sei, was bedeuten würde, dass die Ehefrau von der Beschäftigung ihres Mannes in Deutschland profitiere und ihr daher einen Anspruch auf das Erziehungsgeld zustehe, auch wenn sie nicht in Deutschland wohne. Denn das Erziehungs- oder Elterngeld sei ein Substitut für die verlorene Entlohnung bei Beschäftigung des Elternteils bzw. eine Honorierung für die Erfüllung von Erziehungsarbeit und daher keine abgeleitete, sondern eine selbständige Sicherung, die an den Beschäftigungsort als Einkommensersatzleistung oder an den Wohnsitz anzuknüpfen wäre. Hätte dies der EuGH in seiner Entscheidung Höver/Zachow ebenso gesehen, hätte er sich in der Rechtssache Dodl/Oberhollenzer nicht in eine Rückzugsposition begeben müssen, indem er feststellte, dass das Recht des Nichtwohnstaates eines Elternteils nur subsidiär zur Anwendung komme. Daraufhin entgegnete Kokott, dass bei genauer Beachtung des komplizierten und diffizilen Sekundärrechts, das für den Bereich der Sozialleistungen wie dem Erziehungsgeld in den Verordnungen 1408/71 und 1612/68 geregelt sei, für ein Zurückrudern oder Vorpreschen des EuGH keinerlei Spielraum bestehe. Denn es komme auf eine sorgfältige Analyse der komplexen und in Nuancen dann doch unterschiedlichen Fälle an, die oft zu dem Ergebnis führe, dass die Urteile des EuGH im Ergebnis doch von einer gewissen Konsistenz zeugten und sich der Gerichtshof deshalb nicht in dem monierten Zick-Zack-Kurs bewege. Das Problem liege vielmehr in der Komplexität der zur Entscheidung vorgelegten Sachverhalte. Dem wird allerdings aus den Reihen des Publikums entgegnet, dass man sich immer die Frage stellen müsse, wie in der Sache $H \ddot{o}-$ ver/Zachow zu entscheiden gewesen wäre, wenn das Gemeinschaftsrecht bereits Elterngeld in den Niederlanden gekannt hätte. Dann wäre, weil die Mutter in den Niederlanden lebte, auch die niederländische Leistung gezahlt worden. Dies sei das Ergebnis, das jetzt auch durch die Interpretation des Internationalen Elterngeldrechts durch die Rechtsprechung in der Sache Dodl/Oberhollenzer erreicht worden sei. Zusammenfassend formuliert gehe es darum, dass die Koordination ein hohes Maß an Harmonisierung der Institutionen voraussetze. Sozialleistungssysteme könnten nur dann koordiniert werden, wenn sie in ähnlicher Form in allen Mitgliedstaaten vorhanden bestehen würden. Dieser 